\title{
Multi-Color Emission in Quantum-Dot-Quantum-Well Semiconductor Heteronanocrystals
}

\author{
R. Kostić* And D. Stojanović \\ University of Belgrade, Institute of Physics, Center for Solid State Physics and New Materials \\ P.O. Box 68, 11080 Belgrade, Serbia
}

\begin{abstract}
We present calculation results of multi-color spontaneous emission from quantum-dot-quantum-well semiconductor heteronanocrystals. Our theoretical results explain experimental results of onion-like spherical system similar to: CdSe (core), ZnS (shell), CdSe (shell) spherical quantum dots surrounded by ZnS. We demonstrate influence of shell thickness to exciton localization in distinct layers of heteronanocrystals. Multi-color emission of such heterosystem is determined by $l=0, n=1$ state localization in CdSe core and by $l=0, n=2$ state localization in CdSe shell.
\end{abstract}

PACS numbers: 73.21.La, 78.67.Bf

\section{Introduction}

Recent progress on the synthetic chemistry of semiconductor nanocrystals has made it possible to access high quality semiconductor nanocrystals with controlled size, shape and optical properties. Realization of the dual-emitting 0D-2D nanocrystals indicate that band gap engineering may foster many new quantum structures that can be realized as colloidal nanocrystals. Besides the existing technical applications, ranging from light emitting diodes (LEDs) [1, 2], biomedical labeling [3, 4] or molecular bar-coding [5], these new nanocrystals are perspective for new applications as: spintronics or energy convention.

Multi-color emission in the visible region has been registered in $\mathrm{CdSe}-\mathrm{ZnS}$ based quantum-dot-quantum-well (QDQW) system [6]. Recent experiments indicate that multi-color emitters like (CdSe)ZnS)CdSe (QDQW) heteronanostructures integrated on LEDs hold promise for use as nanoluminophors in future white LEDs (WLEDs) $[7,8]$.

There are still questions about the conditions of multi-color spontaneous emission from such a type of QDQW system. In this paper we present results of our calculations in effective mass approximation, which is widely used method in quantum mechanical analysis. As has been previously shown, the effective mass approximation works well even for few monolayer quantum structures [9-11]. We analyze multi-layered heteronanocrystals of (core)shell)shell)shell structures, in particular case (CdSe) ZnS)CdSe)ZnS.

\section{Model and results}

Nanocrystals such as (core)shell quantum dots typically reveal almost spherical. The single quantum dot we

* corresponding author; e-mail: rkostic@phy.bg.ac.yu consider is spherical heterosystem consisting of a CdSe core with the radius $r_{\mathrm{C}}$, surrounded by $\mathrm{ZnS}$ shell and CdSe shell. We completed this structure with an infinite thick ZnS layer, Fig. 1. The dot center is center of our coordinates.

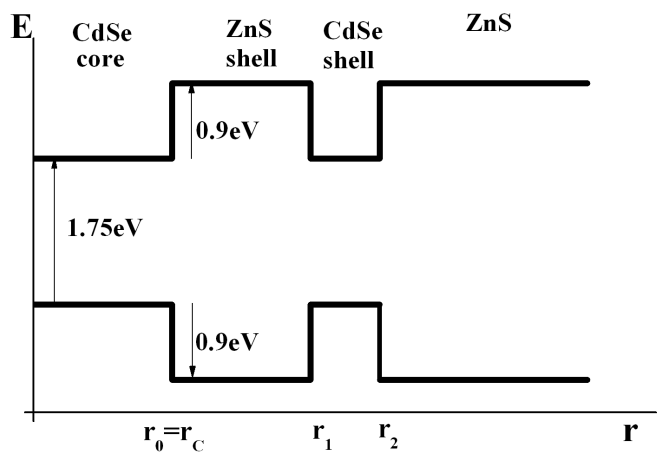

Fig. 1. Radial energy diagram of a (CdSe)ZnS)CdSe)ZnS heteronanocrystal. CdSe core radius is $r_{\mathrm{C}}=r_{0}, \mathrm{ZnS}$ barrier thickness is $r_{1}-r_{0}$ and CdSe shell thickness is $r_{2}-r_{1}$.

Electrons and holes in such a system are characterized by their effective masses and potentials of each region.

Considering that electron and hole spectra are mainly formed by size quantization, the stationary Schrödinger equation for a single particle, in this case, may be expressed as

$$
\left(-\frac{\hbar^{2}}{2 m^{*}} \nabla^{2}+V(r)\right) \Psi(r)=E \Psi(r) .
$$

For spherically symmetric potential $V(r)$ the separation of radial and angular coordinates leads to

$$
\Psi_{n l m}(r)=R_{n l}(r) Y_{l m}(\theta, \varphi) .
$$

$R_{n l}(r)$ is the radial wave function, and $Y_{n l}(\theta, \phi)$ is a spherical harmonic, $n$ is the principal quantum num- 
ber, and $l$ and $m$ are the angular momentum quantum numbers.

For a spherical potential we assumed constant values $V_{0}$ (in CdSe core), $V_{1}$ (in $\mathrm{ZnS}$ shell), $V_{2}=V_{0}$ (in CdSe shell) and $V_{3}=V_{1}$ (in ZnS surrounding material), Fig. 1. In the case when particular energy of heteronanocrystal is higher than the potential in the $i$-th layer, i.e. $E_{n l}>V_{i}$, the corresponding radial wave function $R_{n l}(r)^{i}$ in that layer is a linear combination of spherical Bessel and Neumann functions. In the case $E_{n l}<V_{i}$ corresponding radial wave function $R_{n l}(r)^{i}$ in that layer is a linear combination of the Hankel functions.

The associated wave function solutions must satisfy conditions to be regular when $r=0$, to vanish sufficiently rapidly when $r \rightarrow \infty$, and to satisfy the continuity conditions at all boundaries

$$
\begin{aligned}
& \left.R_{n l}^{i}(r)\right|_{r=r_{i}}=\left.R_{n l}^{i+1}(r)\right|_{r=r_{i}}, \quad i=0, \ldots, N, \\
& \left.\frac{1}{m_{i}^{*}} \frac{\mathrm{d} R_{n l}^{i}(r)}{\mathrm{d} r}\right|_{r=r_{i}}=\left.\frac{1}{m_{i+1}^{*}} \frac{\mathrm{d} R_{n l}^{i+1}(r)}{\mathrm{d} r}\right|_{r=r_{i}}, \\
& \quad i=0, \ldots, N .
\end{aligned}
$$

In this particular example $N=2$ according to the situation that there are four separated regions, i.e. three boundary surfaces. Equations (1) lead to a system of $2 N+2$ linear equations with the $2 N+2$ unknown coefficients. It has nontrivial solutions only if its determinant

$$
D_{l}=D_{l}\left(E_{n l}\right)=0 \text {. }
$$

Once the eigenvalues $E_{n l}$ are determined, the linear equations can be solved yielding the coefficients to be a function of one of them. The last undetermined coefficient is determined by the normalization condition for

$$
R_{n l}(r), \quad \int_{0}^{\infty} R_{n l}^{2}(r) r^{2} \mathrm{~d} r=1 .
$$

Through this procedure we get complete picture of eigensolutions $E_{n l}$ and corresponding wave functions $R_{n l}$. These calculations were performed independently of electrons and holes (procedure is similar), giving the confinement energies $E_{n l}^{\mathrm{e}}$ and $E_{n l}^{\mathrm{h}}$, and corresponding wave functions $R_{n l}^{\mathrm{e}}$ and $R_{n l}^{\mathrm{h}}$.

We focused our analysis to $l=0$ states of zero angular momentum, so called $S$-symmetry, for the first two solutions $n=1,2$. Wavefunction of state $l=0, m=0$ is dependent only on $r$.

Once the electron and hole wave functions are known, radial probability functions $\left(r^{2} R(r)^{2}\right)$ in the system can be obtained to give an illustrative picture of electron and hole spatial localization.

After the electron and hole wave functions are obtained, the Coulomb interaction can be calculated as follows:

$$
E_{\mathrm{C}}=-\mathrm{e}^{2} \iint \frac{\left|R_{\mathrm{e}}\left(r_{\mathrm{e}}\right)\right|^{2}\left|R_{\mathrm{h}}\left(r_{\mathrm{h}}\right)\right|^{2}}{\varepsilon\left(r_{\mathrm{e}}, r_{\mathrm{h}}\right)\left|r_{\mathrm{e}}-r_{\mathrm{h}}\right|} r_{\mathrm{e}}^{2} r_{\mathrm{h}}^{2} \mathrm{~d} r_{\mathrm{e}} \mathrm{d} r_{\mathrm{h}} .
$$

$\varepsilon\left(r_{\mathrm{e}}, r_{\mathrm{h}}\right)$ is the dielectric permittivity.

The electronic transition energy is given by the sum of the CdSe energy gap, corresponding to electron and hole eigenenergies and the Coulomb energy

$$
E_{n l}=E_{g_{\mathrm{CdSe}}}+E_{n l}^{\mathrm{e}}+E_{n l}^{\mathrm{h}}+E_{\mathrm{C}_{n l}} .
$$

All material parameters needed for these calculations are shown in Table $[6,11-13]$. Dimensions of CdSe and $\mathrm{ZnS}$ monolayers (ML), given in Table are assumed as in [6].

TABLE

Material parameters of the system: $E_{\mathrm{g}}$ - energy gap, $\mathrm{CBO}, \mathrm{VBO}$ - conduction and valence band offsets in case CdSe and $\mathrm{ZnS}, m^{*}$ - effective mass, $m_{\mathrm{e}}$ - electron mass, $\varepsilon-$ dielectric permittivity.

\begin{tabular}{c|c|c|c|c|c|c|c}
\hline \hline & $\begin{array}{c}1 \mathrm{ML} \\
{[\mathrm{nm}]}\end{array}$ & $\begin{array}{c}E_{g} \\
{[\mathrm{eV}]}\end{array}$ & $\begin{array}{c}\mathrm{CBO} \\
{[\mathrm{eV}]}\end{array}$ & $\begin{array}{c}\mathrm{VBO} \\
{[\mathrm{eV}]}\end{array}$ & $m_{\mathrm{e}}^{*} / m_{\mathrm{e}}$ & $m_{\mathrm{h}}^{*} / m_{\mathrm{e}}$ & $\varepsilon$ \\
\hline $\mathrm{CdSe}$ & 0.36 & 1.75 & & & 0.13 & 0.45 & 6.1 \\
$\mathrm{ZnS}$ & 0.35 & 3.55 & 0.9 & 0.9 & 0.28 & 0.49 & 8.25
\end{tabular}

We varied CdSe core radius from $1.5 \mathrm{~nm}$ to $3.2 \mathrm{~nm}, \mathrm{ZnS}$ and CdSe shells width from $1 \mathrm{ML}$ to $4 \mathrm{ML}$. In this paper we present only results for $r_{\mathrm{C}}=2.8 \mathrm{~nm}$ and $r_{\mathrm{C}}=1.7 \mathrm{~nm}$ core radii, to illustrate the system behavior.

We start with the analysis of results for $r_{\mathrm{C}}=2.8 \mathrm{~nm}$ heteronanocrystal. We present energies of first two, $n=1$ and $n=2$, electron (upper scale) and hole (lower scale) $l=0$ solutions, Fig. 2. Thickness of the $\mathrm{ZnS}$ barrier does not influence significantly, as presented in Fig. 2. In further analysis we will discuss only results for $4 \mathrm{ML} \mathrm{ZnS}$ shell thickness.

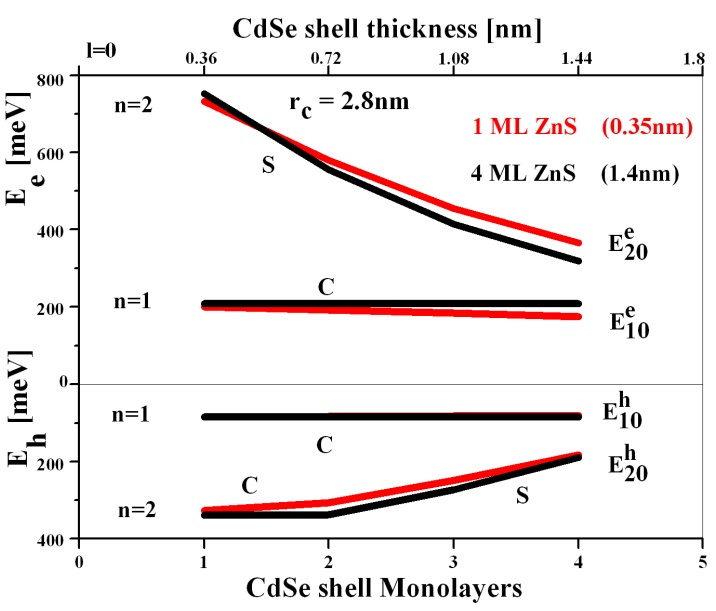

Fig. 2. Electron energies (upper scale) with respect to the bottom of the CdSe conduction band and hole energies (lower scale) according to the top of the CdSe valence band. Core radius is $2.8 \mathrm{~nm}$.

For $n=1$, both electron $\left(E_{10}^{\mathrm{e}}\right)$ and hole $\left(E_{10}^{\mathrm{h}}\right)$ energy as function of CdSe shell thickness are almost unchanged. From radial probability functions, Fig. 3, we can see that both electron and hole are mainly localized in CdSe core, denoted with $\mathrm{C}$ in Fig. 2. It is well known that $\mathrm{ZnS}$ shell efficiently confine excitons in (CdSe)ZnS core-shell nanocrystals [14]. In system under study even 1 ML thick 
ZnS shell makes a good barrier for both the electron and the hole in $n=1$ state. Electron-hole wave function overlaps are close to unity. Exciton binding energy is of the order of $100 \mathrm{meV}$. Calculated $E_{10}$ (Eq. (2)) energy, presented in Fig. 5, is connected to the core dimension and independent of the CdSe shell thickness.

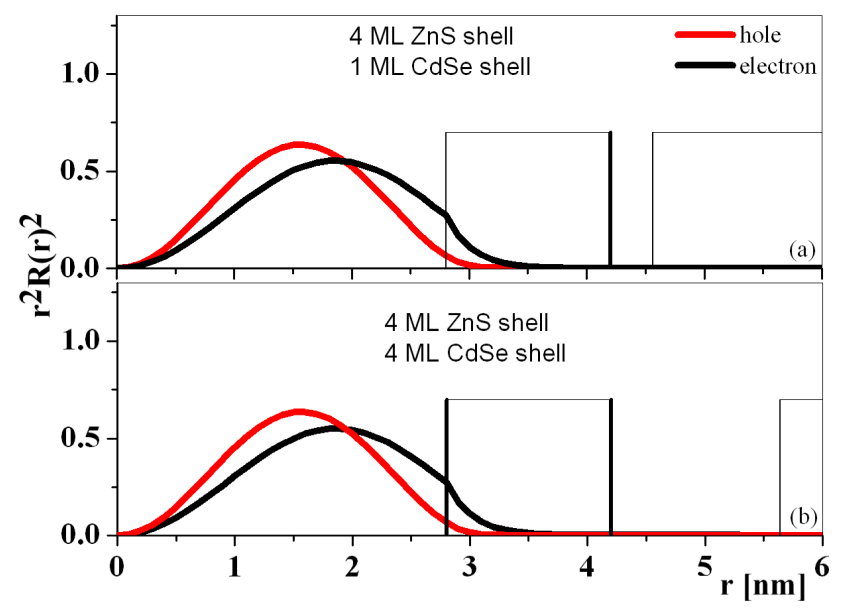

Fig. 3. Radial probability functions for the lowest energy $(l=0, n=1)$ electron and hole wave functions in $r_{\mathrm{C}}=2.8 \mathrm{~nm}$ core radius and $4 \mathrm{ML}(1.4 \mathrm{~nm}) \mathrm{ZnS}$ shell thickness dot: (a) 1 ML CdSe shell thickness; (b) 4 ML CdSe shell thickness.

In case of $n=2$, electron tunnels to the CdSe shell, Fig. 4. For all structures under consideration, presented in Fig. 2, electron is mainly localized in CdSe shell, and calculated energy $E_{20}^{\mathrm{e}}$ depends on the CdSe shell thickness, denoted with $\mathrm{S}$ in Fig. 2. If CdSe shell is thinner than 2 ML: hole is located in the CdSe core; hole slightly tunnels through $\mathrm{ZnS}$ barrier; $E_{20}^{\mathrm{h}}$ is almost constant, Fig. 2 bottom, electron-hole wave function overlaps are small (especially for $\mathrm{ZnS}$ shell wider than $2 \mathrm{ML}$ ); exciton binding energy is of the order of $30 \mathrm{meV}$. Shape of the radial probability function has typical $n=2$ state behavior, Fig. 4a. If CdSe shell is wider than $2 \mathrm{ML}$, hole tunnels to the CdSe shell, Fig. $4 \mathrm{~b} ; E_{20}^{\mathrm{h}}$ changes with the CdSe shell thickness, Fig. 2 bottom. As electron and hole are both localized in the CdSe shell, electron-hole wave function overlap increases. Exciton binding energy is of the order of $20 \mathrm{meV}$. Calculated $E_{20}$ energies are plotted in Fig. 5, upper curve.

In $\left.\left.r_{\mathrm{C}}=2.8 \mathrm{~nm}(\mathrm{CdSe}) \mathrm{ZnS}\right) \mathrm{CdSe}\right) \mathrm{ZnS}$ nanoheterostructure two states are pronounced: lower energy one, connected to the dimension of the CdSe core, and higher energy one connected to the dimension of CdSe shell. Lower energy, core emission, cannot be reabsorbed in the shell. Therefore, in optical spectra of $2.8 \mathrm{~nm}$ core radius two clearly separated features are expected, as experimentally detected in [6].

In Figs. 6 to 9 we present results in the case of core radius $r_{\mathrm{C}}=1.7 \mathrm{~nm}$ and $4 \mathrm{ML} \mathrm{ZnS}$ shell thickness. At $n=1$ states electron energy $E_{10}^{\mathrm{e}}$ is almost constant until $\approx 3 \mathrm{ML}$ CdSe shell and hole energy $E_{10}^{\mathrm{h}}$ until $\approx 4 \mathrm{ML}$

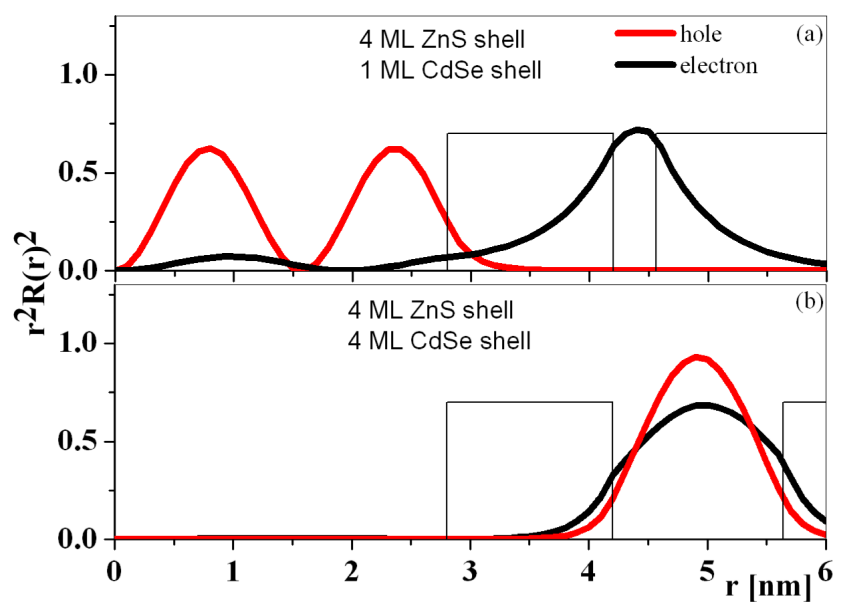

Fig. 4. Radial probability functions for the second solution energy ( $l=0, n=2)$ electron and hole wave functions in $r_{\mathrm{C}}=2.8 \mathrm{~nm}$ core radius and $4 \mathrm{ML}(1.4 \mathrm{~nm})$ ZnS shell thickness dot: (a) 1 ML CdSe shell thickness; (b) 4 ML CdSe shell thickness.

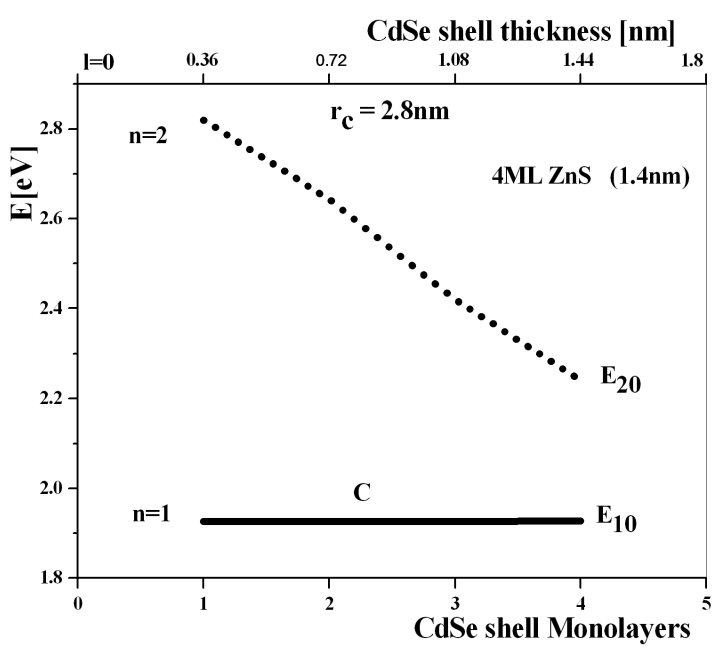

Fig. 5. $E_{10}$ and $E_{20}$ energies for core radius $2.8 \mathrm{~nm}$ and $\mathrm{ZnS}$ shell thickness $4 \mathrm{ML}(1.4 \mathrm{~nm})$. Coulomb interaction is taken into account.

CdSe shell thickness, Fig. 6. From that point both electron and hole energies decrease. Electron is localized in the core for CdSe shell thickness under $3 \mathrm{ML}$, Fig. 7a, and tunnels to the shell if CdSe shell thickness is over $3 \mathrm{ML}$, Fig. 7b. Similar situation is for hole, but critical value is at about 4 ML CdSe shell, Fig. 7.

For $n=2$ state electron energy $E_{20}^{\mathrm{e}}$ decreases until $\approx 3 \mathrm{ML}$ CdSe shell thickness and hole energy $E_{20}^{\mathrm{h}}$ decreases until $\approx 4 \mathrm{ML}$ CdSe shell thickness. After that both energies become practically constant at the values characteristic for $n=1$ state before critical CdSe shell thickness. At state $n=2$ electron is localized in the CdSe shell before critical value, Fig. 8a, and after that in the core, Fig. 8b. Similar change is in hole localization at $\approx 4 \mathrm{ML}$ CdSe shell thickness, Fig. 8 . 


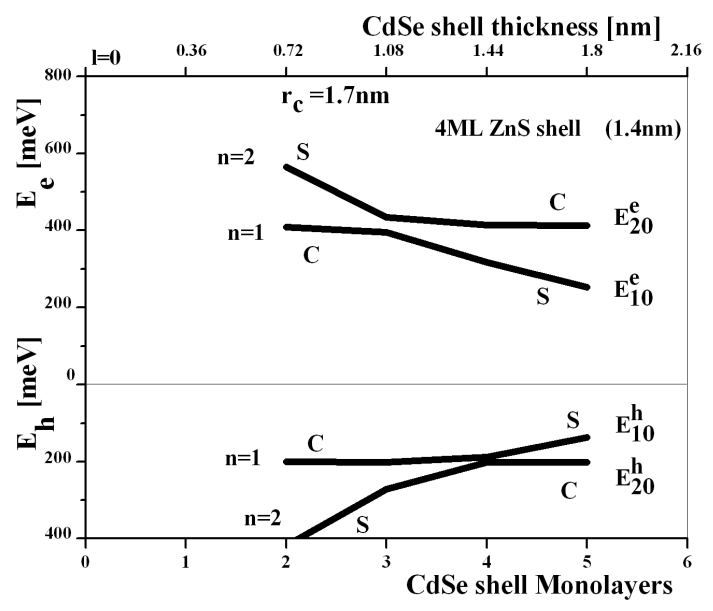

Fig. 6. Electron energies (upper scale) with respect to the bottom of the conduction band CdSe and hole energies (lower scale) according to the top of the CdSe valence band. Core radius is $1.7 \mathrm{~nm}$.

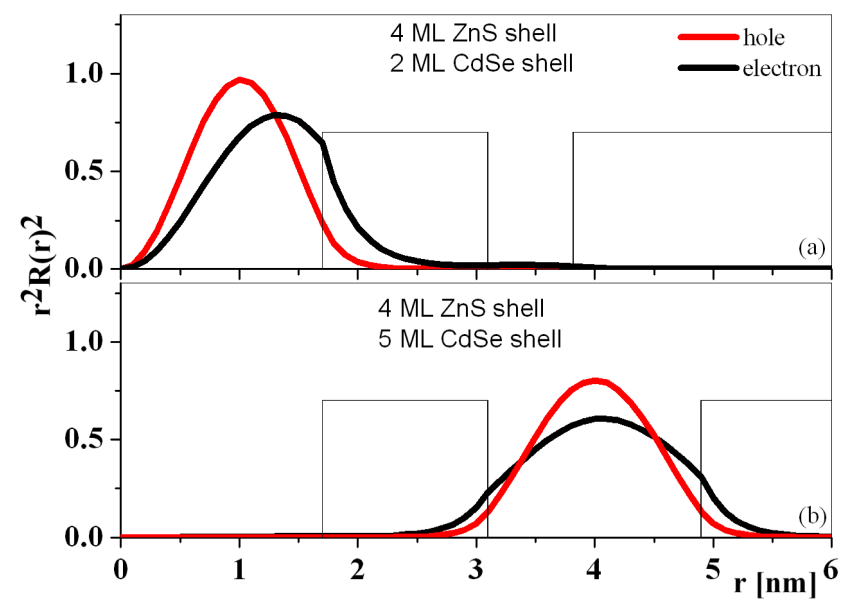

Fig. 7. Radial probability functions for the lowest energy $(l=0, n=1)$ electron and hole wave functions in $r_{\mathrm{C}}=1.7 \mathrm{~nm}$ core radius and $4 \mathrm{ML}(1.4 \mathrm{~nm}) \mathrm{ZnS}$ shell thickness dots: (a) 2 ML CdSe shell thickness; (b) 4 ML CdSe shell thickness.

Exciton binding energy is about $150 \mathrm{meV}$ at $n=1$ state for CdSe shell thickness under $3 \mathrm{ML}$, Fig. 7a, and at $n=2$ state for CdSe shell thickness over 4 ML, Fig. 7b, i.e. when electron and hole are both located in the core. In the opposite case: at $n=1$ state and CdSe shell thickness over $4 \mathrm{ML}$, Fig. $7 \mathrm{~b}$, and at $n=2$ state and CdSe shell thickness is under $3 \mathrm{ML}$, i.e. when electron and hole are both located in the CdSe shell exciton binding energy is less than $20 \mathrm{meV}$. Calculated $E_{20}$ energies are plotted in Fig. 9.

In case $r_{\mathrm{C}}=1.7 \mathrm{~nm}$ and CdSe shell thinner than $3 \mathrm{ML}$ two states are pronounced: lower energy one, connected to the dimension of the CdSe core, and higher energy one connected to the dimension of CdSe shell. Both energies can be identified in optical spectra. For 4 ML CdSe shell,

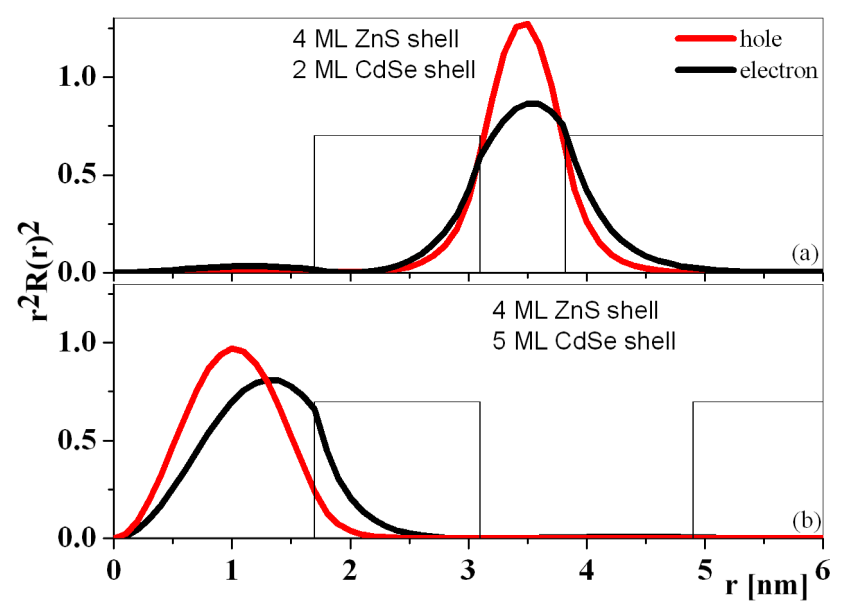

Fig. 8. Radial probability functions for the second solution energy $(l=0, n=2)$ electron and hole wave functions in $r_{\mathrm{C}}=1.7 \mathrm{~nm}$ core radius and $4 \mathrm{ML}(1.4 \mathrm{~nm})$ ZnS shell thickness dots: (a) 1 ML CdSe shell thickness, (b) 4 ML CdSe shell thickness.

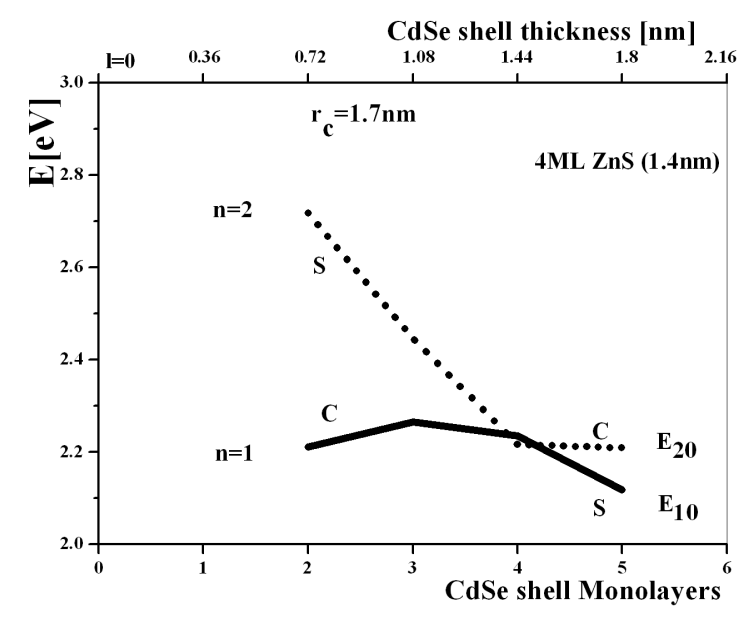

Fig. 9. $\quad E_{10}$ and $E_{20}$ energies for core radius $1.7 \mathrm{~nm}$ and $\mathrm{ZnS}$ shell thickness $4 \mathrm{ML}(1.4 \mathrm{~nm})$. Coulomb interaction is taken into account.

both states are in the same energy region and only one feature in the optical spectra can be registered. If CdSe shell is more than 4 ML thick, higher energy emission from the core can be reabsorbed in the CdSe shell. In optical spectra there is only one feature, i.e. shell emission, as detected in [6].

\section{Conclusion}

We investigated the electronic and optical properties of multi-layered (CdSe)ZnS)CdSe)ZnS nanocrystal and showed that the distinct spatial localization of excitons enable two-color emission.

Energy (wavelength) of the emission that originates from the core is determinated by CdSe core dimension and of the emission that originates from the shell by CdSe 
shell dimension. In some geometry conditions (like $r_{\mathrm{C}}=$ $1.7 \mathrm{~nm}$ and CdSe shell thickness more than $4 \mathrm{ML}$ ) there is no two-color emission and only mono-color emission can be registered.

There are various demands about energies of two-color emission. Two-color emission of nanoparticles that have to be implemented in the solid form for proper integration on a LED platform to generate white light, must be carefully designed to satisfy demands for particular LED platform. Careful analysis and knowledge about basic properties of these nanostructures enables proper design with respect to their applications.

\section{Acknowledgments}

This work is supported by Serbian Ministry of Science, under projects No. 141028 and No. 141047.

\section{References}

[1] M.C. Schlamp, X. Peng, A.P. Alivisatos, J. Appl. Phys. 82, 5837 (1997).

[2] H. Mattoussi, L.H. Radzilowski, B.O. Dabbousi, E.L. Thomas, M.G. Bawendi, M.F. Rubner, J. Appl. Phys. 83, 7965 (1998).

[3] M. Bruchez, M. Moronne, P. Gin, S. Weiss, A.P. Alivisatos, Science 281, 2013 (1998).
[4] W.C.W. Chan, S. Nile, Science 281, 2016 (1998).

[5] M. Han, X. Gao, J.Z. Su, S. Nie, Nat. Biotechnol. 19, 631 (2001).

[6] D Battaglia, B. Blackman, X. Peng, J. Am. Chem. Soc. 127, 10889 (2005).

[7] H.V. Demir, S. Nizamoglu, E. Mutlugun, T. Ozel, S. Sapra, N. Gaponik, A. Eychmuller, Nanotechnology 19, 335203 (2008).

[8] S. Nizamoglu, E. Sari, J. Beak, I. Lee, H.V. Demir, New J. Phys. 10, 123001 (2008).

[9] D. Schoss, A. Mews, A. Eychmuler, H. Weller, Phys. Rev. $\tilde{B}$ 49, 24 (1994).

[10] N.H. Quang, N.T. Truc, Y.M. Niquet, Comp. Mater. Sci. 44, 21 (2008).

[11] S. Nizamoglu, H.V. Demir, Opt. Express 16, 3515 (2008).

[12] A.V. Baranov, Yu. P. Rakovich, J.F. Donegan, T.S. Petrova, R.A. Moore, D.V. Talapin, A.L. Rogach, Y. Masamoto, I. Nabiev, Phys. Rev. B 68, 165306 (2003).

[13] K. Chang, J.B. Xia, Phys. Rev. B 57, 9780 (1988).

[14] D.V. Talapin, A.L. Rogach, A. Kornowski, M. Hasse, H. Weller, Nano Lett. 1, 207 (2001). 